\title{
Apathy is associated with incident dementia in community-dwelling older people
}

Jan Willem van Dalen, MSc, Lennard L. Van Wanrooij, MSc, Eric P. Moll van Charante, MD, PhD, Edo Richard, MD, PhD, and Willem A. van Gool, MD, PhD

Neurology ${ }^{\circledast}$ 2018;90:e82-89. doi:10.1212/WNL.0000000000004767
Correspondence

Dr. van Dalen

j.vandalen@amc.nl

\begin{abstract}
Objective

To assess whether apathy and depressive symptoms are independently associated with incident dementia during 6-year follow-up in a prospective observational population-based cohort study.
\end{abstract}

\section{Methods}

Participants were community-dwelling older people in the Prevention of Dementia by Intensive Vascular Care trial, aged 70-78 years, without dementia at baseline. Apathy and depressive symptoms were measured using the 15-item Geriatric Depression Scale (GDS-15). Dementia during follow-up was established by clinical diagnosis confirmed by an independent outcome adjudication committee. Hazard ratios (HRs) were calculated using Cox regression analyses. Given its potentially strong relation with incipient dementia, the GDS item referring to memory complaints was assessed separately.

\section{Results}

Dementia occurred in 232/3,427 (6.8\%) participants. Apathy symptoms were associated with dementia (HR 1.28, 95\% confidence interval [CI] 1.12-1.45; $p<0.001$ ), also after adjustment for age, sex, Mini-Mental State Examination score, disability, and history of stroke or cardiovascular disease (HR 1.21, 95\% CI 1.06-1.40; $p=0.007$ ), and in participants without depressive symptoms (HR 1.26, 95\% CI 1.06-1.49; $p=0.01$ ). Depressive symptoms were associated with dementia (HR 1.12, 95\% CI 1.05-1.19), also without apathy symptoms (HR 1.16, 95\% CI $1.03-1.31 ; p=0.015)$, but not after full adjustment or after removing the GDS item on memory complaints.

\section{Conclusions}

Apathy and depressive symptoms are independently associated with incident dementia in community-dwelling older people. Subjective memory complaints may play an important role in the association between depressive symptoms and dementia. Our findings suggest apathy symptoms may be prodromal to dementia and might be used in general practice to identify individuals without cognitive impairment at increased risk of dementia. 


\section{Glossary}

CI = confidence interval; CVD = cardiovascular disease; DSM-IV = Diagnostic and Statistical Manual of Mental Disorders, 4th edition; GDS-12D = 12 items of the 15-item Geriatric Depression Scale relating to depression; GDS-15 = 15-item Geriatric Depression Scale; GDS-3A = 3 items of the 15-item Geriatric Depression Scale relating to apathy; HR = hazard ratio; IQR = interquartile range; MMSE $=$ Mini-Mental State Examination.

Apathy is a disorder of motivation, characterized by diminished interest, cognition, and emotional expression, common in community-dwelling elderly. ${ }^{1,2}$ Apathy symptoms can occur both independently and in the context of depression. Several studies have linked symptoms of depression in community-dwelling older people to incident dementia. ${ }^{3}$ These studies, however, did not differentiate between apathy and depressive symptoms. Distinguishing the two is important since they may be independently and differentially associated with dementia. ${ }^{4,5}$

Little is known about the relation between apathy and dementia in community-dwelling older people. In a recent study, a cluster labeled "cognitive/motivational symptoms" that included symptoms of apathy was linked to incident dementia over 3 years in elderly without depression. ${ }^{6}$ Others reported that in otherwise healthy patients with symptomatic small vessel disease, apathy, not depression, was associated with cerebral white matter damage. ${ }^{7}$ Similarly, in mild cognitive impairment, apathy has been associated with progression to dementia, ${ }^{8}$ in some studies contrary to late-life depression. ${ }^{4,5}$ Finally, from a pathophysiologic perspective, apathy is associated with incident vascular disease, which is a risk factor for dementia. ${ }^{8-10}$

We hypothesize that apathy symptoms in community-dwelling older people without dementia are associated with future cognitive decline and dementia independently of depressive symptoms. Furthermore, since memory complaints can occur both as a symptom of depression and as a sign of incipient dementia, we explore how subjective memory complaints influence the relation between depressive symptoms and incident dementia.

\section{Methods}

\section{Participants}

Participants were derived from the Prevention of Dementia by Intensive Vascular Care (PreDIVA) trial. ${ }^{11}$ This trial tested the efficacy of a nurse-led, multicomponent cardiovascular intervention on the prevention of dementia in community-dwelling older persons. Community-dwelling older people (aged 70-78 years) registered with a participating general practice were invited to participate in the trial (figure e-1, http://links.lww.com/ WNL/A11). Exclusion criteria were dementia and disorders likely to hinder successful long-term follow-up (e.g., terminal illness, alcoholism). Recruitment was from June 7, 2006, until March 12, 2009. Follow-up time ranged from 6 years for participants randomized in 2009 up to 8 years for those randomized in 2006-2007. Participants were assessed at baseline and during 2-yearly follow-up assessments. At these visits, data were collected on medical history, medication use, cardiovascular risk factors, cognitive status (Mini-Mental State Examination [MMSE]), depression (15-item Geriatric Depression Scale [GDS-15]), and disability (AMC Linear Disability Scale). The final assessment was conducted on March 4, 2015. The study was approved by the medical ethics committee of the Academic Medical Center, Amsterdam, Netherlands. Participants gave written informed consent before their baseline visit.

\section{Symptoms of apathy and depression}

Symptoms of apathy and depression at baseline were defined corresponding to previous studies. ${ }^{9,10,12,13}$ Symptoms were measured using the GDS-15. Apathy symptoms were operationalized as the 3 apathy items on the GDS-15 (GDS-3A), validated previously. ${ }^{14}$ These items are (1) "Have you dropped many of your activities and interests?" (2) "Do you prefer to stay at home, rather than going out and doing new things?" (3) "Do you feel full of energy?" (reverse-coded). Depressive symptoms were operationalized as the remaining 12 items on the GDS-15 (GDS-12D). Isolated apathy symptoms were operationalized as the GDS-3A score in participants with a score $\leq 1$ on the GDS$12 \mathrm{D}$ and isolated depressive symptoms as the GDS-12D score in participants with a score $\leq 1$ on the GDS-3A. Participants with $>1$ missing item on the GDS-3A or $>2$ items missing on the GDS-12D were excluded from all analyses.

\section{Dementia and cognitive decline}

Dementia was defined as a clinical diagnosis according to DSM-IV criteria, confirmed by 2 members of an independent, blinded outcome adjudication committee based on all available clinical information. Diagnoses of dementia were re-evaluated after 1 year of additional follow-up to avoid falsepositive diagnoses. For participants who dropped out of the study, the dementia status was retrieved by a research nurse from electronic health records or contact with the general practitioner at the end of the study, and presented to the blinded outcome adjudication. The dementia diagnostic process is described in more detail elsewhere. ${ }^{11}$ As secondary endpoint, cognitive decline was operationalized as the change in MMSE score during the study compared to baseline.

\section{Statistical analyses}

Apathy symptoms, isolated apathy symptoms, depressive symptoms, and isolated depressive symptoms at baseline were assessed separately as continuous predictors. Participants with missing data were left out of the analyses. Only baseline symptoms were considered for the main analyses to ensure incident dementia was studied in individuals in whom dementia 
was excluded, and to avoid bias caused by selective drop-out of participants with apathy/depression. Since no relation was expected between baseline symptoms and subsequent random treatment allocation, the intervention and control group were analyzed as a single cohort. A sensitivity analysis adjusting for the intervention was performed to evaluate confounding by treatment allocation. Hazard ratios (HRs) for dementia were calculated using proportional hazard Cox regression. Model 1 adjusted for baseline age and sex; model 2 additionally adjusted for baseline history of cardiovascular disease, history of stroke/ TIA, disability, and MMSE score. The rationale for model 2 was to evaluate whether apathy and depressive symptoms add value to other determinants commonly evaluated in clinical practice for the prediction of dementia. As secondary outcome, we assessed change in MMSE score during the study compared to baseline, using a repeated measurements mixed model adjusted for time to measurement and baseline MMSE score. We explored all covariates for possible interactions with apathy/depressive symptoms in their relation to dementia. To assess the effect of the competing risk of death, we repeated the Cox proportional hazard analyses with mortality and the combined endpoint of dementia or mortality as outcomes. To assess whether there was a dose-effect relationship for the GDS-3A, we assessed HR per step increase on the GDS-3A.

We performed several exploratory analyses: (1) to assess whether apathy/depressive symptoms are associated with dementia hazard in participants with normal to high MMSE scores, we performed analyses in subgroups based on the median baseline MMSE score $\geq 28$ and $<28$; (2) to assess whether apathy/depressive symptoms are associated with short-term rather than long-term development of dementia, as reported for depressive symptoms, ${ }^{15-17}$ we evaluated HRs across tertiles of time to dementia and visually examined whether the dementia survival curves for participants with and without symptoms diverged consistently over time; (3) to analyze whether symptom stability influenced their relation with dementia, we divided participants into 5 categories based on the change in number of symptoms between visits 1 and 2 : increase ( $>1$ higher), decrease ( $>1$ lower), and if not in one of those: stable low (mean $<1$ ), stable moderate (mean 1 through $<2$ ), and stable high $($ mean $\geq 2)$; (4) since the relation between the GDS-12D and incident dementia may be strongly influenced by a single question (GDS-15 number 10) directly referring to subjective memory complaints_- "Do you feel you have more problems with your memory than most people?"- and according to a meta-analysis of the factor structure of the GDS-15 this question may also be considered part of the apathy construct, ${ }^{18}$ we performed a sensitivity analysis with this question removed from the GDS-12D. In these analyses, we defined isolated apathy as apathy in participants with $\leq 1$ symptom on the remaining 11 items on the GDS-12D.

\section{Results}

Characteristics of the study population are reported in table 1. Of the 3,526 participants, $27(0.7 \%)$ were excluded due to missing apathy or depression scores (figure e-1, http://links. lww.com/WNL/A11). These did not differ from those included with regard to baseline age, MMSE score, or proportion developing dementia. At baseline, 693 participants (19.8\%) had $\geq 2$ apathy symptoms, $656(18.7 \%) \geq 2$ depression symptoms, and $322(9.2 \%)$ concurrently $\geq 2$ apathy and $\geq 2$ depression symptoms. Follow-up regarding dementia was complete for 3,427/3,499 (98\%) participants: 232/3,427 (6.8\%) were diagnosed with dementia, a median of 60 months after baseline (interquartile range [IQR] 39-75). Dementia incidence per GDS-3A score and GDS-12D score are illustrated in figure e-2, http://links.lww.com/WNL/A11. In total, 577/3,492 (16.5\%) participants died during follow-up after a median of 54 months (IQR 32-69).

Results of the main analyses are listed in table 2. Adjusted for age and sex, both apathy symptoms (HR per symptom 1.23, 95\% CI 1.08-1.40; $p=0.002$ ) and depressive symptoms (HR 1.11, 95\% CI 1.04-1.19; $p=0.002$ ) were associated with an increased risk of dementia. Associations were similar for isolated apathy (HR 1.20, 95\% CI 1.01-1.43; $p=0.036$ ) and depressive symptoms (HR 1.15, 95\% CI 1.02-1.30; $p=0.018$ ). After additional adjustment for disability, MMSE score, history of cardiovascular disease (CVD), and history of stroke, only apathy (HR 1.21, 95\% CI 1.06-1.40; $p=0.007$ ) and isolated apathy symptoms (HR 1.20, 95\% CI 1.00-1.45; $p=0.046$ ) were associated with an increased risk of dementia. There was an interaction between isolated apathy symptoms and a history of stroke (HR 1.58, 95\% CI 1.01-2.49; $p=0.046$ ), suggesting a stronger association with dementia in this participant group, but this was not significant after Bonferroni correction for the number of tested interactions. Adjusted for age and sex, apathy, isolated apathy, depressive, and isolated depressive symptoms were all associated with decline in MMSE score ( $\beta$ range -0.11 to -0.15 , all $p$ values $<0.001$ ) (table 3 ). Additional adjustment for baseline disability, history of CVD, and history of stroke slightly attenuated these results. There was no significant time by predictor interaction in any of the models.

Sensitivity analyses with additional adjustment for treatment allocation did not meaningfully alter results. In analyses regarding the competing risk of death (tables e- 1 and e-2, http://links.lww.com/WNL/A12), all 4 predictors were associated with a higher risk of death in all models (HR range 1.10 to $1.34, p$ value range $p \leq 0.001$ to $p=0.002$ ). Tables e- 3 through e-6 list results according to the number of apathy symptoms. There was no clear dose-response relationship between the number of apathy symptoms and dementia risk. Decline in MMSE score during the study did increase with more apathy symptoms. There was also a cumulative relation between the number of apathy symptoms and mortality (figure 1). Results were generally similar for isolated apathy. 
Table 1 Population characteristics of the whole population and in apathy and depression subgroups

\begin{tabular}{|c|c|c|c|c|c|}
\hline & Total & $\begin{array}{l}\geq 2 \text { Apathy } \\
\text { symptoms }\end{array}$ & $\begin{array}{l}\geq 2 \text { Depressive } \\
\text { symptoms }\end{array}$ & $\begin{array}{l}\geq 2 \text { Apathy symptoms, } \\
<2 \text { depressive symptoms }\end{array}$ & $\begin{array}{l}\geq 2 \text { Depressive symptoms, } \\
<2 \text { apathy symptoms }\end{array}$ \\
\hline Total n (\%) & 3,499 & $693(19.8)$ & $656(18.7)$ & 371 & 334 \\
\hline Age, $y$, mean \pm SD & $74.3 \pm 2.5$ & $74.8 \pm 2.4$ & $74.5 \pm 2.4$ & $74.9 \pm 2.5$ & $74.4 \pm 2.4$ \\
\hline Female sex, n (\%) & $1,899 / 3,499(54.3)$ & 415/693 (59.9) & 403/656 (61.4) & 212/371 (57.1) & 200/334 (59.9) \\
\hline $\begin{array}{l}\text { ALDS score, mean } \pm \\
\text { SD }\end{array}$ & $87 \pm 5.1$ & $83.7 \pm 8$ & $84 \pm 8.5$ & $85 \pm 5.3$ & $86 \pm 6.1$ \\
\hline MMSE score & $28(27-29)$ & $28(27-29)$ & $28(27-29)$ & $28(27-29)$ & $28(27-29)$ \\
\hline History of CVD, n (\%) & $1,033 / 3,476(29.7)$ & 259/688 (37.6) & 235/653 (36) & 137/369 (37.1) & 113/334 (33.8) \\
\hline $\begin{array}{l}\text { History of stroke/TIA, } \\
n(\%)\end{array}$ & $345 / 3,453(10)$ & $121 / 680(17.8)$ & 117/647 (18.1) & $56 / 363(15.1)$ & $52 / 330(15.8)$ \\
\hline GDS-15 score & $1(0-2)$ & $4(3-6)$ & $4(3-6)$ & $3(2-3)$ & $3(2-4)$ \\
\hline GDS-15 score >4, n (\%) & $326 / 3,499(9.3)$ & 261/693 (37.7) & $326 / 656$ (49.7) & 0/371 (0.0) & 65/334 (19.5) \\
\hline $\begin{array}{l}\text { Apathy symptoms } \\
\text { (GDS-3A) }\end{array}$ & $0(0-1)$ & $2(2-3)$ & $1(1-2)$ & $2(2-2)$ & $1(0-1)$ \\
\hline $\begin{array}{l}>1 \text { Apathy symptom, } \\
\text { n (\%) }\end{array}$ & 693/3,499 (19.8) & 693/693 (100) & $322 / 656(49.1)$ & $371 / 371(100)$ & 0/334 (0.0) \\
\hline $\begin{array}{l}\text { Depression } \\
\text { symptoms (GDS-12D) }\end{array}$ & $0(0-1)$ & $1(0-3)$ & $3(2-4)$ & $0(0-1)$ & $2(2-3)$ \\
\hline $\begin{array}{l}>1 \text { Depression } \\
\text { symptom, } \mathrm{n}(\%)\end{array}$ & $656 / 3,499(18.7)$ & $322 / 693$ (46.5) & $656 / 656(100)$ & 0/371 (0.0) & $334 / 334(100)$ \\
\hline $\begin{array}{l}\text { Dementia incidence } \\
\text { rate (per } 1,000 \\
\text { person-years) }\end{array}$ & 10.7 & 13.7 & 14.5 & 12.1 & 10.7 \\
\hline
\end{tabular}

Abbreviations: ALDS = AMC linear disability scale (higher scores represent higher capability of disability); CVD = cardiovascular disease, including myocardial infarction, angina pectoris, and peripheral arterial disease; GDS-12D = 12 items of the 15 -item Geriatric Depression Scale relating to depression; GDS-15 = 15item Geriatric Depression Scale; GDS-3A = 3 items of the 15-item Geriatric Depression Scale relating to apathy; MMSE = Mini-Mental State Examination. Apathy symptoms defined as $>1$ symptom on the 3 GDS-3A; depressive symptoms defined as $>1$ symptom on the GDS-12D; isolated apathy symptoms: symptoms of apathy without symptoms of depression; isolated depressive symptoms: symptoms of depression without symptoms of apathy.

Results in groups with baseline MMSE score $\geq 28$ or $<28$ are listed in tables e-7 and e-8 (http://links.lww.com/WNL/ A12). Compared to our main results, associations with dementia were less strong in the group with an MMSE score $<28$, and not significant. In participants with an MMSE score $\geq 28$, adjusted for age and sex, apathy symptoms (HR 1.26, 95\% CI 1.04-1.51; $p=0.016)$ and isolated apathy symptoms (HR 1.27, 95\% CI 1.01-1.61; $p=0.041$ ) were associated with incident dementia. These associations were slightly attenuated in model 2, leaving none significant. Results for the

Table 2 Association between predictors at baseline and incident dementia

\begin{tabular}{|c|c|c|c|c|c|c|c|c|c|c|c|c|}
\hline & \multicolumn{4}{|c|}{ Unadjusted } & \multicolumn{4}{|c|}{ Model 1} & \multicolumn{4}{|c|}{ Model 2} \\
\hline & Event/total & $\mathrm{HR}$ & $95 \% \mathrm{Cl}$ & $p$ Value & Event/total & HR & $95 \% \mathrm{Cl}$ & $p$ Value & Event/total & $\mathrm{HR}$ & $95 \% \mathrm{Cl}$ & $p$ Value \\
\hline Apathy & $232 / 3,425$ & 1.28 & $1.12-1.45$ & $<0.001$ & $232 / 3,425$ & 1.23 & $1.08-1.40$ & 0.002 & $224 / 3,343$ & 1.21 & $1.06-1.40$ & 0.007 \\
\hline Isolated apathy & $175 / 2,786$ & 1.26 & $1.06-1.49$ & 0.010 & $175 / 2,786$ & 1.20 & $1.01-1.43$ & 0.036 & $170 / 2,718$ & 1.20 & $1.00-1.45$ & 0.046 \\
\hline Depression & $232 / 3,425$ & 1.12 & $1.05-1.19$ & 0.001 & $232 / 3,425$ & 1.11 & $1.04-1.19$ & 0.002 & $224 / 3,343$ & 1.07 & $0.99-1.15$ & 0.102 \\
\hline Isolated depression & $176 / 2,737$ & 1.16 & $1.03-1.31$ & 0.015 & $176 / 2,737$ & 1.15 & $1.02-1.30$ & 0.018 & $170 / 2,678$ & 1.08 & $0.95-1.22$ & 0.268 \\
\hline
\end{tabular}

Abbreviations: $\mathrm{Cl}$ = confidence interval; $\mathrm{HR}=$ hazard ratio.

Model 1: crude; model 2: adjusted for age and sex; model 3: additionally adjusted for disability (AMC linear disability scale), MMSE score, history of cardiovascular disease, and history of stroke. Symptoms measured using the 15-item Geriatric Depression Scale (GDS-15). Apathy symptoms: score on the 3 apathy items on the GDS-15 (GDS-3A); depressive symptoms: score on the remaining 12 items on the GDS-15 (GDS-12D); isolated apathy: GDS-3A score in participants with a score $\leq 1$ on the GDS-12D; isolated depressive symptoms: GDS-12D score in participants with a score $\leq 1$ on the GDS-3A. 
analyses divided according to tertiles of time to dementia are listed in table e-9 (http://links.lww.com/WNL/A12). The associations seem to be strongest for all predictors for incident dementia $<3.89$ years, compared to dementia between 3.89 and 5.77 years and $>5.77$ years after baseline. However, the divergence between dementia survival curves for participants with and without apathy symptoms at baseline was constant over time (figure 2). Similarly, the survival curves for depressive symptoms did not show any clear changes in the association between depressive symptoms and dementia over time. Longitudinal results regarding the change in apathy/ depressive symptoms from visits 1 to 2 are listed in tables e-10 and e-12. Participants in the decreasing number of symptoms category had lower HRs compared to those in the increasing, stable moderate, and stable high categories, but group sizes were small. Results for the sensitivity analysis removing GDS item 10- "Do you feel you have more problems with your memory then most people?" - are listed in table e-10. In these analyses, the number of depressive symptoms on the remaining 11 items of the GDS-12D were not associated with dementia (HR 1.06, 95\% CI 0.97-1.15; $p=0.192$ ). The relation between isolated apathy and incident dementia was slightly stronger (model 1: HR 1.22, 95\% CI 1.04-1.44; $p<$ 0.016 ; model 2: HR 1.22, 95\% CI 1.03-1.46; $p=0.022$ ).

\section{Discussion}

Our results suggest symptoms of apathy are associated with incident dementia in community-dwelling older people without cognitive impairment. This association is independent of depressive symptoms, age, sex, MMSE score, disability, and history of CVD or stroke. Depressive symptoms were also associated with dementia independent of age and sex but no longer when the symptom of subjective memory complaints was left out. Both apathy and depressive symptoms are independently associated with cognitive decline according to the MMSE, independent of age, sex, baseline MMSE score, disability, and history of CVD or stroke. The associations may be stronger for short-term $(<4$ years) compared to long-term dementia incidence and attenuated in patients with remitting symptoms.

The association of depressive symptoms in older people with cognitive decline and dementia has been reported previously. ${ }^{19-22}$ However, these studies did not differentiate among apathy, subjective memory complaints, and other depressive symptoms. The association of apathy with cognitive decline has not been described previously in unselected community-dwelling older people but corresponds to findings in mild cognitive impairment and other neurologic disease. ${ }^{4,5,23,24}$ Furthermore, a recent study reported that a symptom cluster labeled "cognitive/motivational symptoms," which included symptoms of apathy, predicted incident dementia over 3 years in elderly without depression. ${ }^{6}$ Findings in patients with mild cognitive impairment that apathy is associated with future cognitive decline while depression is not ${ }^{4,5}$ were not corroborated by our study, 


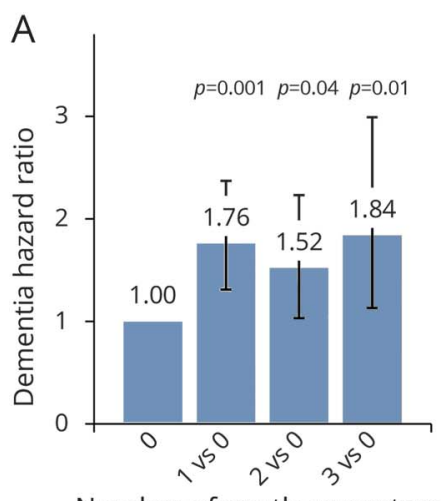

Number of apathy symptoms
B

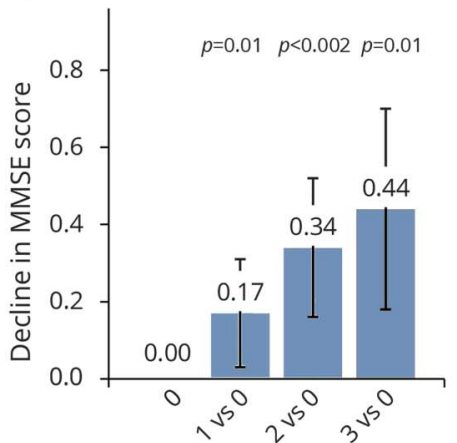

Number of apathy symptoms

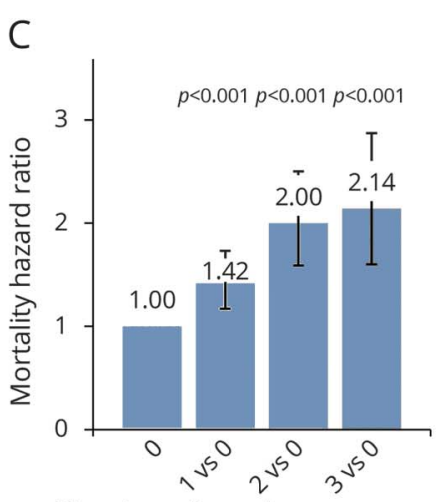

Number of apathy symptoms

Depicted are hazard ratios and $p$ values for (A) dementia and (C) mortality, and (B) adjusted mean decline in Mini-Mental State Examination (MMSE) score (higher value equals greater decline) during the study, for 1, 2, and 3 apathy symptoms compared to 0 symptoms, adjusted for age and sex. Symptoms measured using the 15-item Geriatric Depression Scale (GDS-15). Apathy symptom categories are based on 0, 1, 2, or 3 symptoms on the 3 apathy items of the GDS-15; as comparison, depressive symptoms are based on $0,1,2$, or $\geq 3$ symptoms on the remaining 12 items of the GDS-15.

although the association with incident dementia was stronger for apathy symptoms, and for depressive symptoms seemed largely dependent on the symptom of memory complaints. This last finding was unexpected and could suggest that dysphoric symptoms of depression are not related to future cognitive decline while symptoms of apathy are. However, given the exploratory nature of this finding and the limited scope of the GDS-15, our study does not provide enough evidence to warrant this conclusion and requires affirmation by future studies. The absence of a dose-response relationship between apathy symptoms and dementia risk is contrary to reports regarding incident cardiovascular disease. ${ }^{12}$ This absence may be related to the strong doseresponse relation between the number of apathy symptoms and the competing risk of death. In participants with high apathy scores, mortality may occur before dementia can develop. Our findings suggesting that apathy and depressive symptoms are more related to development of dementia in the short term rather than the long term agree with previous reports on depressive symptoms and dementia. ${ }^{15-17}$ They

Figure 2 Proportion of participants in study without dementia over time for participants with and without apathy symptoms

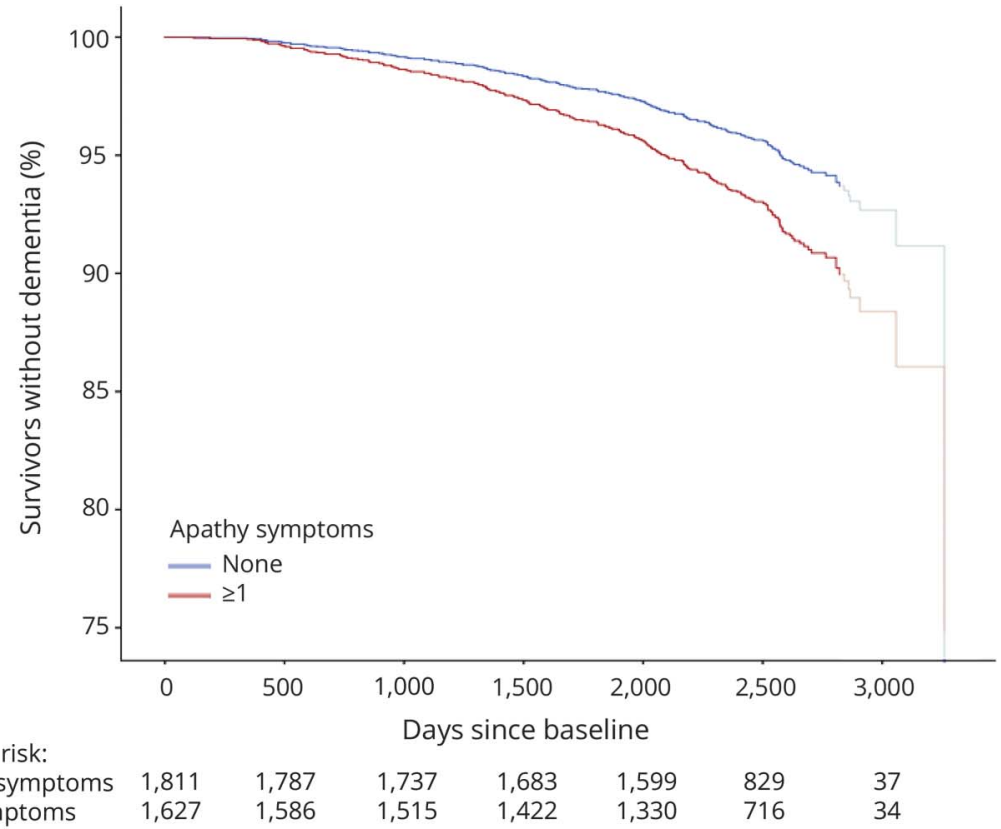

Survival plot for dementia for participants with and without apathy symptoms, adjusted for age, sex, disability, Mini-Mental State Examination score, history of cardiovascular disease, and history of stroke/TIA. Participants who were deceased or finished the study are censored at the time of death or final assessment. The curve is shaded from the 90th percentile of days since baseline (2,829 days). Hazard ratio (95\% confidence interval) across tertiles of follow-up time: $<1,421$ days: 1.32 (1.04-1.68); 1,421-2,107 days: $1.10(0.86-1.41) ;>2,107$ days: 1.30 (1.01-1.67). 
could indicate that apathy and depressive symptoms are an early marker of dementia, rather than a true risk factor. However, we found no clear decline of HRs across tertiles of time to dementia, and the divergence between the dementia survival curves seemed relatively constant. Our results therefore remain inconclusive. The finding that patients with decreasing symptom scores have a lower risk of dementia is concordant with previous results in depression. ${ }^{21}$ These findings may however be affected by small group numbers and requires more in-depth analyses in larger studies to allow for any conclusions. Due to the risk of selective drop-out, the effect sizes in these analyses cannot be compared directly to those of the main analyses.

This study has some limitations. Individuals deemed unlikely to be able to complete 6 years of follow-up due to a medical condition were excluded from participating. This may have affected the distribution of apathy and depressive symptoms in our cohort and influenced their associations with dementia incidence. Results regarding the dose-response relation between apathy symptoms and dementia should be interpreted with caution due to the relatively small number of dementia cases and participants endorsing all apathy symptoms, especially for isolated apathy. The narrow age window at baseline (70-78 years) may have left us unable to detect any interactions between apathy/depressive symptoms and age in their relation with dementia. Selective drop-out may have influenced the reliability of our analyses regarding decline in MMSE score, since MMSE scores were unavailable from the time participants discontinued the study. However, our main analysis with all-cause dementia as outcome was not hampered by selective drop-out, with $98 \%$ complete follow-up for this outcome. Finally, it is important to stress that the depressive symptoms referred to in this article do not include apathy symptoms and therefore do not represent the overall construct of depression as it is currently diagnosed, which includes symptoms of apathy.

In our study, community-dwelling older people without manifest cognitive impairment endorsing all GDS-3A apathy symptoms had approximately double the hazard of dementia compared to those endorsing none, independent of age, disability, and MMSE score. Subgroup analyses imply the hazard is higher in patients with normal to high MMSE scores ( $>27$ points). The more than twofold increase in mortality risk further illustrates the clinical relevance of apathy symptoms in these persons. Our population-based sample, its similarity to national (cohort) data, ${ }^{25}$ and the completeness of follow-up on all-cause dementia (98.0\%) and mortality (99.8\%) suggest our results are generalizable. The factor structure of the GDS-15 and the likelihood of individuals endorsing these symptoms may vary across cultures and translations. ${ }^{18}$ Our findings require validation and further exploration, preferably in datasets with more dementia cases, longer follow-up, detailed apathy and depression assessment, and broader age range. The mechanism relating apathy to dementia is unknown. Conceivably, apathy is a behavioral manifestation of subclinical cerebral atherosclerosis, cerebrovascular brain damage (e.g., small vessel disease), or other pathology associated with cognitive decline and dementia. ${ }^{7,26}$ This could be assessed in largescale longitudinal studies involving brain MRI.

This study contributes to the accumulating evidence stressing the relevance of recognizing apathy in the general older population, regardless of concurrent depressive symptoms. , $^{70,12,26,27}$ Given their independent association with incident dementia and mortality, clinicians should be watchful of apathy symptoms, although their possibly limited specificity must be considered when evaluating future risk of dementia. Distinguishing apathy from depression is important. Although depression is a relatively well-known risk factor for health deterioration in old age, persons with apathy symptoms without dysphoric symptoms may easily be overlooked, especially since they may tend to withdraw from care. Since depressive symptoms other than memory complaints did not seem to be related to dementia risk, apathy symptoms may be better suited to alert general practitioners to vulnerable persons who could benefit from a more active caregiving approach. If replicated, the differential associations for apathy and depressive symptoms suggest that studies regarding the relation between depression and dementia must distinguish the 2 to allow comprehensive interpretation. In research, our findings suggest apathy symptoms may be prodromal to dementia and may be useful to consider when trying to identify persons at increased risk.

\section{Author contributions}

J.W.v.D.: study design, analysis and interpretation of the data, drafting of the manuscript. L.L.V.W.: critical revision of the manuscript. E.P.M.v.C.: study concept, critical revision of the manuscript. E.R.: study concept, critical revision of the manuscript. W.A.v.G.: study concept and design, analysis and interpretation of the data, critical revision of the manuscript.

\section{Acknowledgment}

The authors thank the participants of the PreDIVA study and everyone involved in the study's conduction.

\section{Study funding}

The PreDIVA trial was supported by the Dutch Ministry of Health, Welfare and sport (grant 50-50110-98-020), the Dutch Innovation Fund of Collaborative Health Insurances (grant 05-234), and the Netherlands Organisation for Health Research and Development (grant 62000015). The funders had no role in study design, data collection and analysis, decision to publish, or preparation of the manuscript.

\section{Disclosure}

The authors report no disclosures relevant to the manuscript. Go to Neurology.org/N for full disclosures.

Received May 15, 2017. Accepted in final form September 27, 2017.

\section{References}

1. Ishii S, Weintraub N, Mervis JR. Apathy: a common psychiatric syndrome in the elderly. J Am Med Dir Assoc 2009;10:381-393. 
2. Marin RS. Apathy: a neuropsychiatric syndrome. J Neuropsychiatry Clin Neurosci 1991;3:243-254

3. Diniz BS, Butters MA, Albert SM, Dew MA, Reynolds CF. Late-life depression and risk of vascular dementia and Alzheimer's disease: systematic review and meta-analysis of community-based cohort studies. Br J Psychiatry 2013;202:329-335.

4. Palmer K, Di Iulio F, Varsi AE, et al. Neuropsychiatric predictors of progression from amnestic-mild cognitive impairment to Alzheimer's disease: the role of depression and apathy. J Alzheimers Dis 2010;20:175-183.

5. Richard E, Schmand B, Eikelenboom P, et al. Symptoms of apathy are associated with progression from mild cognitive impairment to Alzheimer's disease in non-depressed subjects. Dement Geriatr Cogn Disord 2012;33:204-209.

6. Lugtenburg A, Zuidersma M, Voshaar RCO, Schoevers RA. Symptom dimensions of depression and 3-year incidence of dementia: results from the Amsterdam study of the elderly. J Geriatr Psychiatry Neurol 2015;29:99-107.

7. Hollocks MJ, Lawrence AJ, Brookes RL, et al. Differential relationships between apathy and depression with white matter microstructural changes and functional outcomes. Brain 2015;138:3803-3815.

8. Belleville S, Fouquet C, Duchesne S, Collins DL, Hudon C. Detecting early preclinical Alzheimer's disease via cognition, neuropsychiatry, and neuroimaging: qualitative review and recommendations for testing. J Alzheimers Dis 2014;42(suppl 4): S375-S382.

9. Ligthart SA, Richard E, Fransen NL, et al. Association of vascular factors with apathy in community-dwelling elderly individuals. Arch Gen Psychiatry 2012;69: 636-642.

10. van der Mast RC, Vinkers DJ, Stek ML, et al. Vascular disease and apathy in old age: The Leiden 85-Plus Study. Int J Geriatr Psychiatry 2008;23:266-271.

11. Moll van Charante EP, Richard E, Eurelings LS, et al. Effectiveness of a 6-year multidomain vascular care intervention to prevent dementia (preDIVA): a clusterrandomised controlled trial. Lancet 2016;388:797-805.

12. Eurelings LSM, Ligthart SA, van Dalen JW, Moll van Charante EP, van Gool WA, Richard E. Apathy is an independent risk factor for incident cardiovascular disease in the older individual: a population-based cohort study. Int J Geriatr Psychiatry 2014; 29:454-463.

13. Mitchell J, Mathews HF, Yesavage JA. A multidimensional examination of depression among the elderly. Res Aging 1993;15:198-219.
14. Bertens AS, Moonen JEF, de Waal MWM, et al. Validity of the three apathy items of the Geriatric Depression Scale (GDS-3A) in measuring apathy in older persons. Int J Geriatr Psychiatry 2017;32:421-428.

15. Mirza SS, de Bruijn RFAG, Direk N, et al. Depressive symptoms predict incident dementia during short- but not long-term follow-up period. Alzheimers Dement 2014;10:S323-S329.

16. Lenoir H, Dufouil C, Auriacombe S, et al. Depression history, depressive symptoms, and incident dementia: the 3C study. J Alzheimers Dis 2011;26:27-38.

17. Richard E, Reitz C, Honig LH, et al. Late-life depression, mild cognitive impairment, and dementia. JAMA Neurol 2013;70:374-382.

18. Kim G, DeCoster J, Huang C-H, Bryant AN. A meta-analysis of the factor structure of the Geriatric Depression Scale (GDS): the effects of language. Int Psychogeriatr 2013;25:71-81.

19. Rosenberg PB, Mielke MM, Xue QL, Carlson MC. Depressive symptoms predict incident cognitive impairment in cognitive healthy older women. Am J Geriatr Psychiatry $2010 ; 18: 204-211$.

20. Verdelho A, Madureira S, Moleiro C, et al. Depressive symptoms predict cognitive decline and dementia in older people independently of cerebral white matter changes: the LADIS study. J Neurol Neurosurg Psychiatry 2013;84:1250-1254.

21. Mirza SS, Wolters FJ, Swanson SA, et al. 10-year trajectories of depressive symptoms and risk of dementia: a population-based study. Lancet Psychiatry 2016;3:628-635.

22. Kaup AR, Byers AL, Falvey C, et al. Trajectories of depressive symptoms in older adults and risk of dementia. JAMA Psychiatry 2016;73:525-531.

23. Brodaty H, Liu Z, Withall A, Sachdev PS. The longitudinal course of post-stroke apathy over five years. J Neuropsychiatry Clin Neurosci 2013;25:283-291.

24. Dujardin K, Sockeel P, Delliaux M, Destée A, Defebvre L. Apathy may herald cognitive decline and dementia in Parkinson's disease. Mov Disord 2009;24:2391-2397.

25. Ligthart SA, Richard E, van Gool WA, et al. Cardiovascular risk management in community-dwelling elderly: opportunities for prevention. Eur J Prev Cardiol 2012; 19:1365-1372.

26. Grool AM, Geerlings MI, Sigurdsson S, et al. Structural MRI correlates of apathy symptoms in older persons without dementia: AGES-Reykjavik Study. Neurology 2014;82:1628-1635.

27. Groeneweg-Koolhoven I, de Waal MWM, van der Weele GM, Gussekloo J, van der Mast RC. Quality of life in community-dwelling older persons with apathy. Am J Geriatr Psychiatry 2014;22:186-194. 


\section{Apathy is associated with incident dementia in community-dwelling older people}

Jan Willem van Dalen, MSc, Lennard L. Van Wanrooij, MSc, Eric P. Moll van Charante, MD, PhD, Edo Richard, MD, PhD, and Willem A. van Gool, MD, PhD

Neurology 2018;90:27. doi:10.1212/WNL.0000000000004767
Correspondence

Dr. Willem van Dalen j.vandalen@amc.nl

\section{Study question}

Are apathy and depressive symptoms independently associated with incident dementia in community-dwelling elderly people?

\section{Summary answer}

Apathy and depressive symptoms are independently associated with incident dementia in community-dwelling elderly people.

\section{What is known and what this paper adds}

Apathy is associated with depressive symptoms, which are in turn associated with incident dementia. This study provides evidence that apathy itself is independently associated with dementia.

\section{Participants and setting}

This study involved 3,427 community-dwelling Dutch older persons (age 70-78 years) who were recruited between June 7, 2006, and March 12, 2009, for the PreDIVA trial.

\section{Design, size, and duration}

All participants underwent baseline assessments and follow-up assessments at 2-year intervals for 6-8 years. These assessments included measurements of apathy and depressive symptoms via the 15-item Geriatric Depression Scale (GDS-15).

\section{Primary outcomes}

Dementia diagnoses were based on the criteria in the $\mathrm{Di}$ agnostic and Statistical Manual of Mental Disorders, Fourth Edition. These diagnoses were confirmed by a blinded adjudication committee and re-evaluated at 1-year follow-up timepoints to avoid false-positives.

\section{Main results and the role of chance}

Over the study period, 232 (6.8\%) participants were diagnosed with dementia. Age- and sex-adjusted proportional hazard Cox regression models showed that apathy (hazard ratio [HR] per symptom $1.23,95 \%$ CI 1.08-1.40; $p=0.002)$ and depressive symptoms (HR 1.11, 95\% CI 1.04-1.19; $p=0.002$ ) were associated with dementia. Similar results were obtained in analyses of apathy in persons without depressive symptoms (HR 1.20, 95\% CI 1.01-1.43; $p=0.036$ ) and depressive symptoms in persons without apathy (HR 1.15, 95\% CI $1.02-1.30 ; p=0.018$ ).

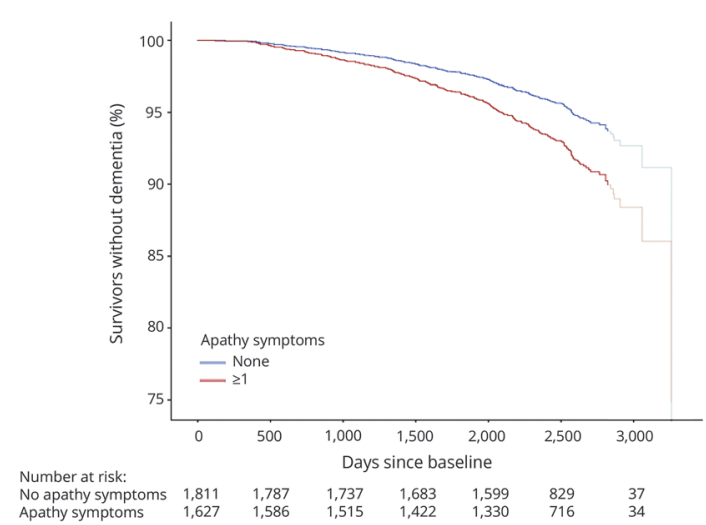

When memory symptoms were not included as depressive symptoms, depressive symptoms did not predict dementia.

\section{Bias, confounding, and other reasons for caution}

The GDS-15 has a limited scope. Relatively few participants were diagnosed with dementia. The participants' narrow age range may have hindered analyses of how apathy and depressive symptoms interacted with age in their effects on dementia. The study's definition of depressive symptoms excluded apathy symptoms, but apathy symptoms are currently considered part of the depression construct.

When memory symptoms were not included as depressive symptom, depressive symptoms did not predict dementia.

\section{Generalizability to other populations}

This study excluded individuals deemed unlikely to complete 6 years of follow-up because of a comorbid medical condition, so the results may not be generalizable to such individuals.

\section{Study funding/potential competing interests}

The study was funded by the Dutch government, the Dutch health insurance industry, and the Netherlands Organisation for Health Research and Development. Go to Neurology.org/N for full disclosures. 


\section{Neurology}

Apathy is associated with incident dementia in community-dwelling older people Jan Willem van Dalen, Lennard L. Van Wanrooij, Eric P. Moll van Charante, et al. Neurology 2018;90;e82-e89 Published Online before print December 1, 2017

DOI 10.1212/WNL.0000000000004767

This information is current as of December 1, 2017

\section{Updated Information \& Services}

References

Citations

Subspecialty Collections

Permissions \& Licensing

Reprints including high resolution figures, can be found at: http://n.neurology.org/content/90/1/e82.full

This article cites 27 articles, 3 of which you can access for free at: http://n.neurology.org/content/90/1/e82.full\#ref-list-1

This article has been cited by 2 HighWire-hosted articles: http://n.neurology.org/content/90/1/e82.full\#\#otherarticles

This article, along with others on similar topics, appears in the following collection(s):

All Cognitive Disorders/Dementia

http://n.neurology.org/cgi/collection/all_cognitive_disorders_dementia All Neuropsychology/Behavior http://n.neurology.org/cgi/collection/all_neuropsychology_behavior All Psychiatric disorders

http://n.neurology.org/cgi/collection/all_psychiatric_disorders Depression

http://n.neurology.org/cgi/collection/depression

Information about reproducing this article in parts (figures,tables) or in its entirety can be found online at:

http://www.neurology.org/about/about_the_journal\#permissions

Information about ordering reprints can be found online:

http://n.neurology.org/subscribers/advertise

Neurology ${ }^{\circledR}$ is the official journal of the American Academy of Neurology. Published continuously since 1951, it is now a weekly with 48 issues per year. Copyright Copyright @ 2017 The Author(s). Published by Wolters Kluwer Health, Inc. on behalf of the American Academy of Neurology.. All rights reserved. Print ISSN: 0028-3878. Online ISSN: 1526-632X.

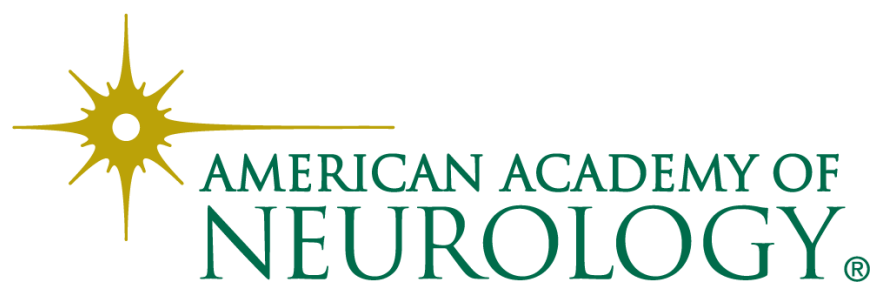

